\title{
The Belle II experiment: Status and prospects
}

\author{
Alberto Martini ${ }^{1,2, *}$ \\ ${ }^{1}$ Dipartimento di Matematica e Fisica, Università degli Studi Roma Tre, Via della Vasca Navale, 84 \\ Rome 00146, Italy \\ ${ }^{2}$ INFN Sezione di Roma Tre, Via della Vasca Navale, 84 Rome 00146, Italy
}

\begin{abstract}
This article aims to describe the Belle II experiment, its status and physics prospects for the next several years. Belle II is situated in Japan, at the KEK laboratory and it is the upgraded version of the Belle experiment. It uses a new collider named SuperKEKB, a second generation of B-factory based on the innovative Nano-Beam scheme technique, which is expected to collect an integrated luminosity of $50 \mathrm{ab}^{-1}$. Using this amount of data, together with improved detector performances, it will be possible to provide important contributions about several flavour physics topics (i.e. CKM matrix elements, FCNC processes, quarkonium states etc..) through high precision measurements. The main aim of Belle II is to investigate new physics scenarios and validate highly suppressed SM predictions. The experiment is almost completely assembled; it already took the first data without the vertex detector installed while the data taking will start in February 2019.
\end{abstract}

\section{Introduction}

An essential role in the understanding of the Standard Model (SM) and its mechanisms is played by the heavy flavour physics. In the past, experiments at B-factories investigated deeply this field (BaBar using PEP II and Belle using KEKB) producing important discoveries concerning the $B$ meson physics and more. So far many SM predictions have still to be verified and the investigation of New Physics (NP) processes is crucial. For those reasons a second generation of B-factories is rising together with a corresponding experiment. That experiment is Belle II which relies on an international collaboration composed of more than 700 researchers from 25 countries and it takes advantage of the largest Japanese laboratory for particle physics KEK, in Tsukuba. The new SuperKEKB collider [1] (Fig. 1 left), together with an improved detector system with respect to its predecessor Belle, have been assembled and tested at KEK, and it plays a key role in the investigation of NP.

Thanks to several upgrades, Belle II will collect data up to a total integrated luminosity of $50 \mathrm{ab}^{-1}$ and will take under control the increased background coming from the higher instant luminosity $\mathcal{L}_{\text {max }}=8 \cdot 10^{35} \mathrm{~cm}^{-2} \mathrm{~s}^{-1}$ (Fig. 1 right). The whole dataset will allow to investigate several highly suppressed processes and some of them will be introduced later (see Ref. [2] for more details). Furthermore, the low-background environment provided by the B-factory, w.r.t. an hadronic machine, will produce large data samples of $\mathrm{B}, \mathrm{D}, \tau$ giving exclusive advantages to the experiment.

The time-line of Belle II can be divided into three main phases (Fig. 2):

*e-mail: alberto.martini@roma3.infn.it,alberto.martini@uniroma3.it 

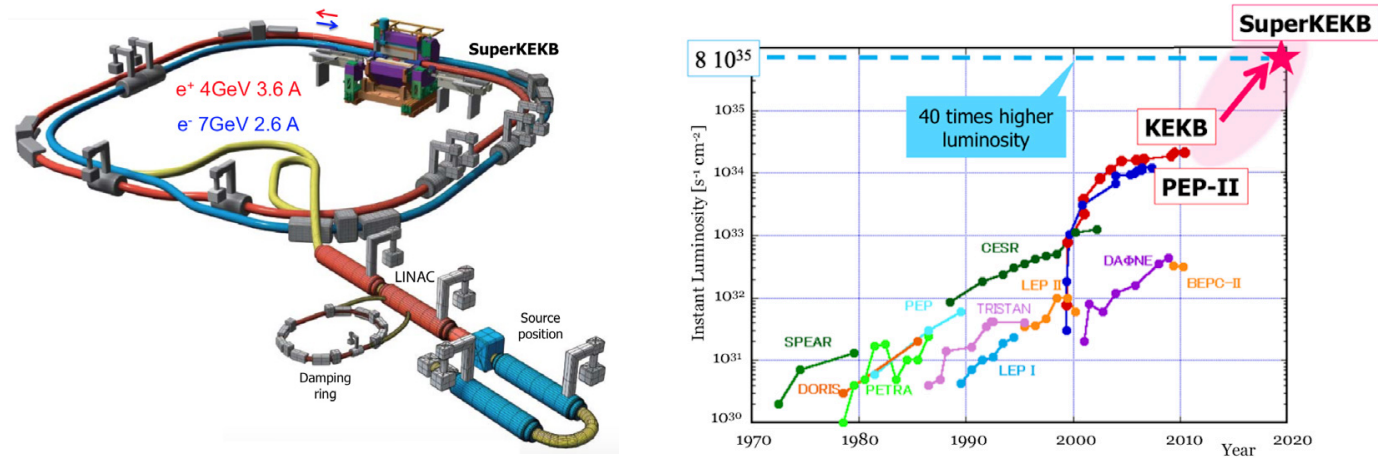

Figure 1. SupeKEKB schematic view (left) and luminosity comparison between SuperKEKB and colliders of the past (right).

- Phase 1: it has been successfully completed in 2016. It concerned the commissioning of SuperKEKB;

- Phase 2: it ended in July 2018 and some physics data have been collected without the silicon vertex detector installed. Those data will provide some first physics results, mainly in the dark sector field, even with partial detector installed.

- Phase 3: it will start in February 2019 and it consists in the data taking with the whole detector installed.

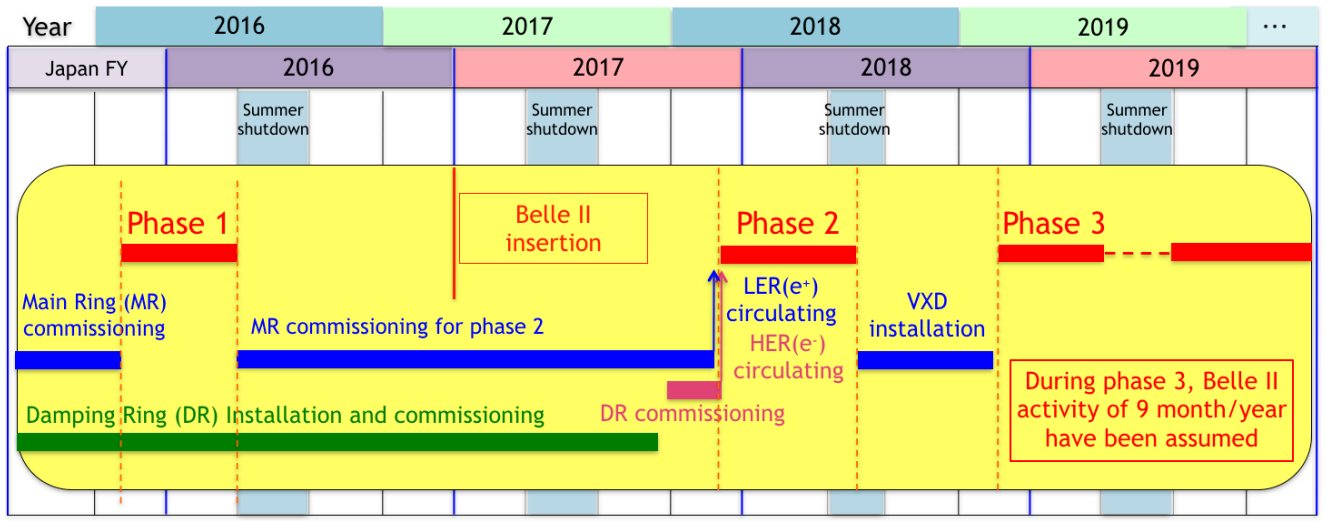

Figure 2. Status and future plan scheme of the experiment.

\section{Collider and detector}

SuperKEKB uses asymmetric electron and positron beams resulting in a boost of the center of mass (CM) system corresponding to a factor $\beta \gamma=0.28$ in order to enable time-dependent $\mathrm{CP}$ violation measurements. The CM energy will be just above the threshold of the $\Upsilon(4 S)$ 
resonance production which predominantly decays into $B \bar{B}$ couples. In order to increase the statistics collected by the experiment, SuperKEKB uses an innovative technique named Nano-Beam scheme, originally proposed for the Italian SuperB project by P. Raimondi [3], which will allow to reach 40 times higher luminosity w.r.t. KEKB. The luminosity of the machine can be written as follows:

$$
\mathcal{L}=\frac{\gamma_{ \pm}}{2 e r_{e}}\left(1+\frac{\sigma_{y}^{*}}{\sigma_{x}^{*}}\right) \frac{I_{ \pm} \xi_{y \pm} R_{L}}{\beta_{y_{ \pm}} R_{\xi_{y}}}
$$

where $r_{e}$, e and $\gamma$ are respectively the electron classical radius, the elementary electric charge and the Lorentz factor. The \pm signs distinguish the positron (+) from the electron (-) while the ratio between the parameters $R_{L}$ and $R_{\xi_{y}}$ represents a geometrical reduction factor. The overall value of the parameters just mentioned is around 1 so the luminosity depends on the remaining parameters: the total beam current $I_{ \pm}$(increased by a factor $\sim 2$ ), the vertical beambeam parameter $\xi_{y \pm}$ (slightly increased) and the vertical beta function at the IP $\beta_{y \pm}^{*}$ (decreased by a factor $\sim 20$ ). The Nano-Beam scheme allows to squeeze the beams at the interaction point up to $\sim 50 \mathrm{~nm}$ among y and $\sim 5 \mu \mathrm{m}$ along $\mathrm{x}$, as shown in Fig. 3. As a consequence the machine background increased thus, in order to compensate this effect, the Belle II detector has to be improved.

Belle

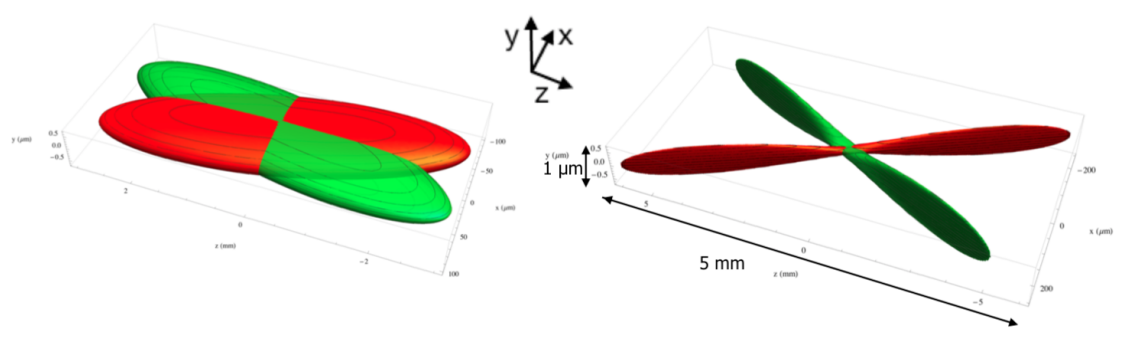

Figure 3. The Nano-Beam scheme improvements w.r.t. Belle.

The detector (Fig. 4), starting from the interaction point, is made of [4]:

- Vertex detector (VXD): it is composed by 2 layers of completely new Pixel vertex Detector (PXD) and 4 layers of fast Silicon-strip Vertex Detector (SVD). With respect to the vertex detector used by Belle, the VXD is faster and will increase the vertex resolution of a factor $\sim 2$;

- Central Drift Chamber (CDC): it is the main tracking detector with an improved resolution thanks to smaller cell size;

- Particles identification system: it is made of the Time-Of-Propagation counter (TOP) placed in the barrel region and the Aerogel Ring Imaging Cherenkov detector (ARICH) placed in the forward end-cap region. Their main aim is to distinguish pions from kaons.

- Electromagnetic Calorimeter (ECL): it is substantially the same of the one used for the Belle detector, with a faster read-out electronics; 
- $K_{L}$ and $\mu$ detector (KLM): it has been improved by substituting all the Resistive Plate Chamber (RPC) layers with scintillators in the end-caps region and the first 2 layers in the barrel region.

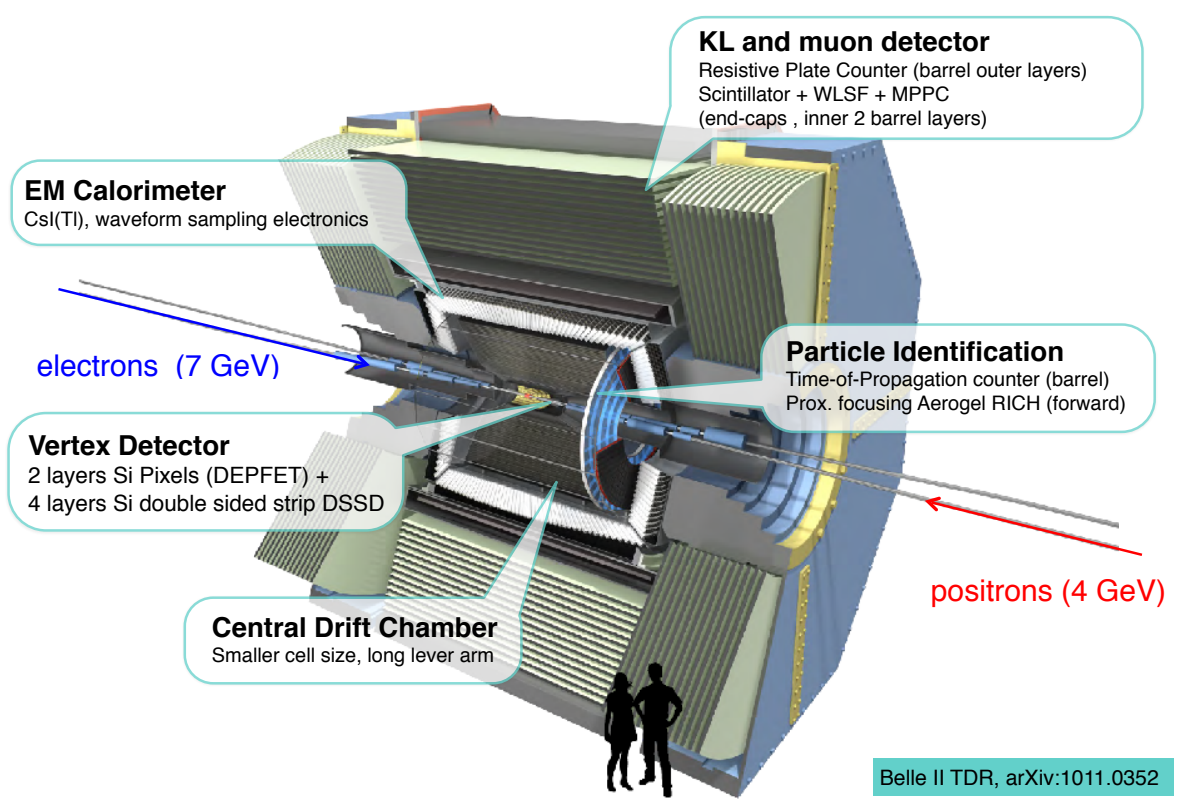

Figure 4. Schematic view of the Belle II detector.

\subsection{Physics program}

The experiment aims to investigate with high precision several heavy flavour physics fields as a B-factory [2]. The detector together with the collider will provide important advantages in the context of a physics analysis. SuperKEKB will produce coherent couples of B mesons from $\mathrm{Y}(4 \mathrm{~S})$ resonance in a clean environment w.r.t. experiments at hadronic machines and large data samples of B, D and $\tau$ with low background will be collected. This is the substantial difference between Belle II and its main competitor LHCb whose results are very important to make as much comparisons as possible. A valuable aspect is that, combining the results of both experiments it is possible to cover almost every heavy flavour physics field. Another important characteristic of the Belle II detector is a good hermeticity (around $90 \%$ of the whole solid angle) which, together with good reconstruction efficiency and resolution for neutral particles as $\eta^{0}, K^{0}$ and $\pi^{0}$, will provide important advantages for decays with missing energy. Moreover, Belle II takes advantage of a powerful tool called Full Event Interpretation (FEI) [5]. It consists in the complete reconstruction of the B-tag side in both semi-leptonic (more efficient but less precise) and hadronic (more precise but less efficient) ways in order to obtain the flavour of the B-tag. Thus it is possible to infer the flavour of the B-signal and isolate particles coming from it, having large advantages in analysis with missing energy and 
missing mass (Fig. 5). The FEI algorithm can be exploited because of the background-clean

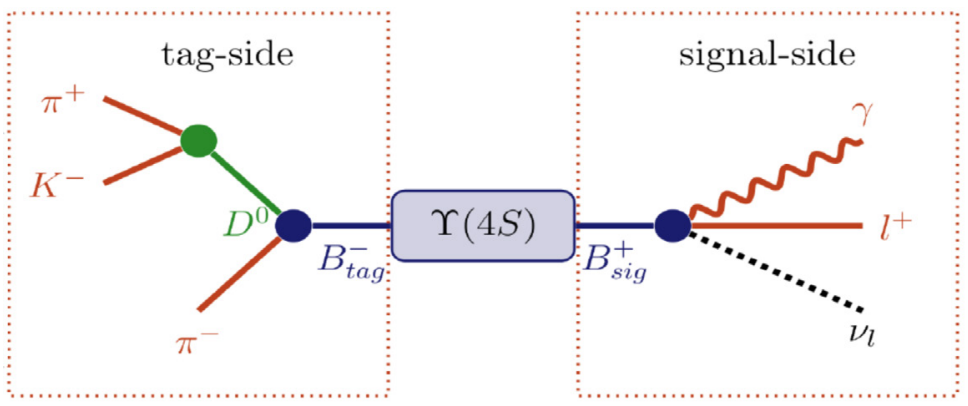

Figure 5. Schematic example of the FEI algorithm mechanism in a particular case.

environment and the very good hermeticity of the detector. It will provide a greater efficiency, w.r.t. previous versions of the algorithm, yielding a larger effective sample size usable in the measurement.

Taking advantages of the exclusive features just mentioned, part of the Belle II physics program can be summarized as below:

- Unitarity Triangle (UT) angles and CKM matrix elements (Fig. 6 left): CP violating measurements (time-dependent and time-integrated) allow to discover new possible CPviolating phases that indicates the existence of SM extensions;

- Dark sector: search for dark matter candidates i.e. dark photon (Fig. 6 right). Belle II will use a dedicated single photon trigger in order to be able to reconstruct its decay into an invisible final state (the signature is the presence of a single photon and missing energy). Some results can be obtained before the beginning of Phase 3.
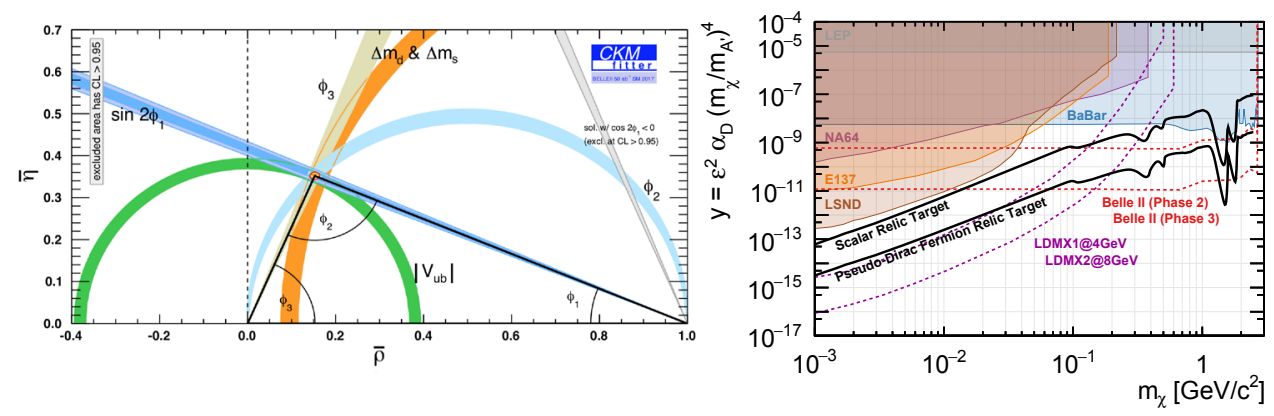

Figure 6. Expected UT constraints that can be obtained with the whole statistic of Belle II (left) and limits on the coupling vs the mass of the dark candidate (right).

- Flavour Changing Neutral Current (FCNC): penguin processes described by quark transitions like $b \rightarrow s$ and mixing processes of neutral meson states allow to search for NP in loops;

- Leptonic decays and Lepton Flavour Violation (LFV): study of $\tau$ and leptonic $B$ decays in order to probe NP scenarios which take into account NP models i.e. extended Higgs sector or right-handed neutrino couplings. LFV can be investigated mainly thanks to the clean environment provided by a B-factory which makes Belle II highly competitive (Fig. 7); 


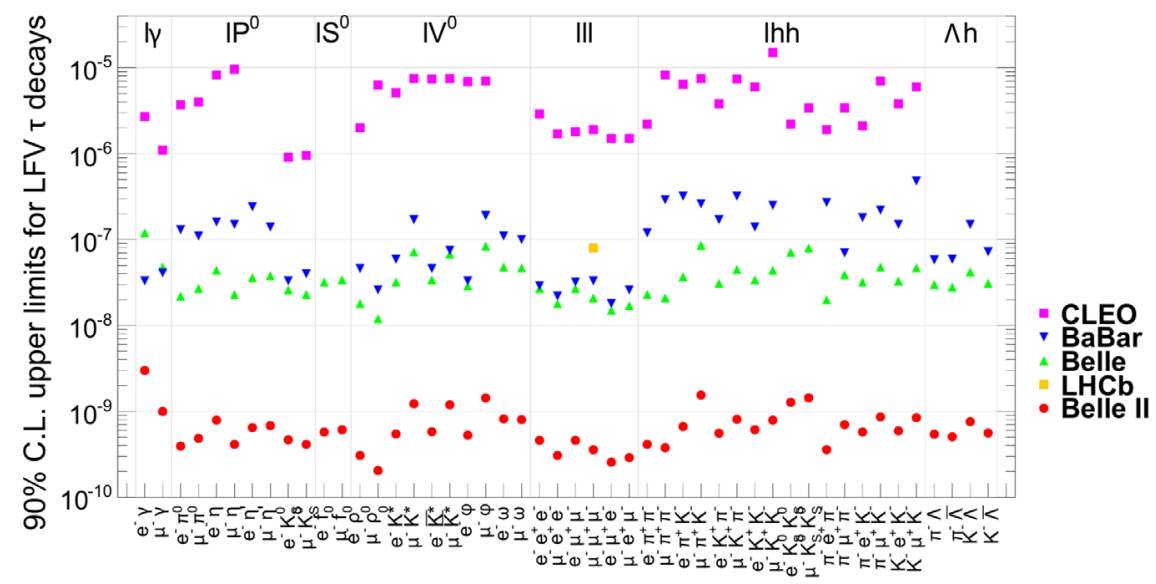

Figure 7. Expected upper limits on the LFV in several $\tau$ decays [2].

- Hadronic spectroscopy and quarkonium: a different center of mass energy of the collider is needed in order to produce resonances like $\Upsilon(3 S), \Upsilon(5 S)$ and $\Upsilon(6 S)$ allowing to study several intermediate bounded states and their properties. Quarkonium can be produced in different ways, some of them accessible only by a B-factory like the two-photon interaction and the double charmonium production. Currently there are many predicted intermediate states to be investigated and Belle II can provide the needed sensitivity to do that.

Using a part of the Phase 2 data, Belle II has been able to obtain several known results like the $K_{S}$ mass reconstruction as shown in Fig. 8 left. It is also important to underline that the instant luminosity reached during Phase 2 was of $\sim 10^{33} \mathrm{~cm}^{-2} \mathrm{~s}^{-1}$, obtained by squeezing the beams up to a value of $\beta$ of $6 \mathrm{~mm}$ along y direction at the interaction point (Fig. 8 right).
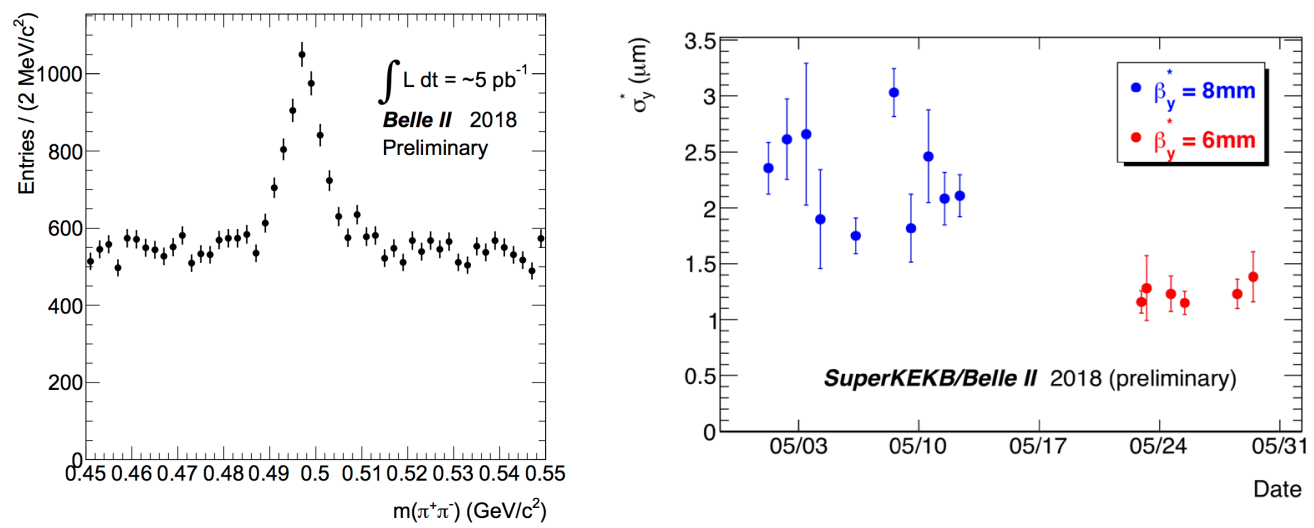

Figure 8. $K_{S}$ mass distribution (left) and vertical beam size at the interaction point vs time (right). 


\subsection{Conclusions}

The Belle II experiment expected to collect an huge dataset of $50 \mathrm{ab}^{-1}$ which will largely extend our knowledge of flavour physics. The experiment successfully completed the accelerator commissioning and it already took some data with partial detector installed while the data taking will begin on February 2019. With the first collected data, Belle II already produced some known results and first physics studies are ongoing, mainly in the dark sector field.

The physics program of the experiment is wide. Belle II will be competitive not only in the B-physics field but also in the D and $\tau$ physics, as it will provide clean datasets w.r.t. experiments at hadron colliders. Belle II aims to search for NP signals and most of the sensitivity studies have already been performed by the collaboration [2].

\section{References}

[1] Y.Ohnishietal et al. 2013, Accelerator design at SuperKEKB, Prog. Theor. Exp. Phys. 03A011, 17

[2] E. Kou et al., The Belle II Physics book, 2018, arXiv:1808.10567 [hep-ex].

[3] P. Raimondi, M. Zobov and D. Shatilov, Proceedings of EPAC08, Genoa, Italy, 2620 (2008).

[4] Abe T et al. 2010, Belle II Technical Design Report, arXiv:1011.0352, 480

[5] T. Keck et al., The Full Event Interpretation - An exclusive tagging algorithm for the Belle II experiment, arXiv:1807.08680 [hep-ex]. 